“ (C) 2017 IEEE. Personal use of this material is permitted. Permission from IEEE must be obtained for all other uses, in any current or future media, including

reprinting/republishing this material for advertising or promotional purposes, creating new collective works, for resale or redistribution to servers or lists, or reuse of any copyrighted component of this work in other works." 


\title{
Signal Stripping Based Sensing Parameter Estimation in Perceptive Mobile Networks
}

\author{
J. Andrew Zhang, Xiaojing Huang, Y. Jay Guo and Md. Lushanur Rahman*
}

\begin{abstract}
We propose signal stripping based approaches for estimating detailed channel composition parameters for sensing in the recently proposed perceptive mobile networks where simultaneous communication and radar sensing is performed. Via reconstructing a (differentiated) channel matrix, we obtain a signal model which can be solved by conventional compressive sensing (CS) techniques. Parameter estimation methods based on a 1-D CS algorithm are proposed and their effectiveness is validated by provided simulation results.
\end{abstract}

\section{INTRODUCTION}

The recently proposed perceptive mobile network [1] can enable simultaneous communication and radio sensing in cellular networks using joint communication and sensing techniques [2]. It can potentially provide seamless radio sensing wherever there is mobile signal coverage, for detecting, tracking, and identifying objects, activities and events, while providing non-compromised communication services.

Extraction of sensing parameters in mobile networks is very challenging due to complicated mobile signals. A modern mobile network is a complex heterogeneous network, connecting diverse devices that occupy staggered resources interleaved over time, frequency and space. Most existing techniques for communication, radar and localization are incapable of operating directly in this environment. For example, communication only requires to estimate composited coefficients instead of detailed channel compositions, radar systems generally use optimized or unmodulated transmitted signals, and localization mainly solves the problem of finding the first multipath signal.

Complex propagation environment with a lot of clutter is another major challenge for sensing in preceptive mobile networks. Base-stations (BSs) typically see numerous reflected signals, and a lot of them are clutters from static background environment and may not be required for sensing. Existing clutter suppression techniques in Radar are not efficient here because they mainly deal with small environmental clutters, thanks to both the working

\footnotetext{
*University of Technology Sydney, Global Big Data Technologies Centre (GBDTC), Sydney, Australia, e-mail: \{Andrew.Zhang; Xiaojing.Huang; Jay.Guo\}@uts.edu.au; MdLushanur.Rahman@student .uts.edu.au
}

environment and the use of narrow beamforming to control the scanning direction and zones.

In this paper, we propose a signal stripping based approach, combined with compressive sensing technique, to overcome these two major challenges. This approach uses the estimated data symbols and channels to strip signals from different nodes and keep as few as a single nodes signal or its estimated composited channel for sensing at a time. This is achieved by refining the composited channel estimation in communication, and then reconstructing and subtracting the clutter to be removed. A 1-D compressive sensing algorithm is then proposed to estimating sensing parameters such as signal propagation delay, Doppler shift, and angle of departure and arrival. This scheme can significantly reduce the number of sensing parameters to be estimated, reduce the algorithm complexity and improve its performance, compared to the block compressive sensing algorithm in [1]. Numerical results are provided and verify the effectiveness of the proposed scheme.

\section{SIGNAL MODELS AND PROBLEM FORMULATION}

Similar to [1], we consider a typical network where $Q$ BSs work cooperatively to provide connections to $K$ mobile users, using multiuser MIMO techniques. Each BS and user have an $M$-element and $M_{T^{-}}$ element linear antenna arrays, respectively. For both uplink and downlink, we assume that MIMOOFDMA type of modulation is applied. Let $N$ denote the total number of subcarriers and $B$ the total bandwidth. Then the subcarrier interval is $f_{0}=$ $B / N$ and OFDM symbol period is $T_{s}=N / B+T_{p}$ where $T_{p}$ is the period of cyclic prefix. Let $\mathcal{S}$ denote the actual set of subcarriers that are used for a link, and denote the number of used subcarriers as $N_{u}$

Assume a planar wave-front in signal propagation. The array response vector of a size- $M$ linear array is given by

$$
\mathbf{a}(M, \theta)=\left[1, e^{j \kappa \sin (\theta)}, \cdots, e^{j \kappa(M-1) \sin (\theta)}\right]^{T},
$$

where $\kappa=2 \pi d / \lambda, d$ is antenna interval in the array, $\lambda$ is the wavelength, and $\theta$ is either angle-of-depart (AoD) or angle-of-arrival (AoA). 
In [1], we defined uplink and downlink sensing, i.e., direct, and passive and active sensing, as sensing at a BS using uplink signals from mobile users and downlink signals from itself and other cooperative BSs, respectively. We also show that they can have a common mathematical formulation. Hence without loss of generality, we present our scheme here by referring to uplink sensing.

At a BS, the received and processed discrete signal at the $n$-th subcarrier and the $t$-th OFDM symbol can be represented as

$$
\mathbf{y}_{n, t}=\sum_{k=1}^{K} \mathbf{H}_{n, k, t} \mathbf{x}_{n, k, t}+\mathbf{z}_{n, t} .
$$

where $\mathbf{x}_{n, k, t}$ are the transmitted signals at subcarrier $n$ from the $k$-th user, $\mathbf{z}_{n, t}$ is AWGN, and $\mathbf{H}_{n, k, t}$ is the $M \times M_{T}$ time-varying uplink channel matrix between user $k$ and the BS

$$
\begin{array}{r}
\mathbf{H}_{n, k, t}=\sum_{\ell=1}^{L_{k}} b_{k, t, \ell} e^{-j 2 \pi n \tau_{k, t, \ell} f_{0}} e^{j 2 \pi t f_{D, k, t, \ell} T_{s} .} \\
\mathbf{a}\left(M, \phi_{k, t, \ell}\right) \mathbf{a}^{T}\left(M_{T}, \theta_{k, t, \ell}\right) .
\end{array}
$$

In (3), for the $\ell$-th multipath, $b_{k, t, \ell}$ is its amplitude, $\tau_{k, t, \ell}$ is the propagation delay, $\theta_{k, t, \ell}$ and $\phi_{k, t, \ell}$ are the AoD and AoA, respectively, and $f_{D, k, t, \ell}$ is the associated Doppler frequency. The channel is assumed to be quasi-static, which means that all the parameters remain unchanged over the period of several OFDM symbols.

Now the basic task for sensing is to estimate sensing parameters $\left\{\tau_{k, t, \ell}, f_{D, k, t, \ell}, \phi_{k, t, \ell}, \theta_{k, t, \ell}, b_{k, t, \ell}\right\}$ from the received signals. For communications, we are more interested in the composited values of $\mathbf{H}_{n, k, t}$, instead of the channel details.

\section{SIGNAL STRIPPING BASED SENS- ING PARAMETER ESTIMATION}

\subsection{Signal Stripping}

Based on (2), a block compressive-sensing scheme was developed in [1] to extract sensing parameters. However, the approach has very high complexity due to the signal block structure.

The idea of signal stripping is to simplify the basic signal structure by separating signals and channels for different users and removing the modulated symbols from the signal from $\mathbf{y}_{n, t}$. Channel matrices in communications are generally estimated with the assistance of interpolation techniques and hence its accuracy is insufficient for estimating sensing parameters. Since sensing can tolerate much larger processing delay than communications, we can exploit the demodulated signals in communication to develop a signal stripping based sensing scheme. A communication packet generally lasts a few milliseconds. We can use decision directed channel estimation (DDCE) [3] technology to reconstruct the channel matrix for each user individually, and do sensing based on the reconstructed channel. Different to conventional DDCE algorithms applied for communications, we only need to reconstruct channels as accurate as possible, but do not need to do channel prediction. Detailed reconstruction algorithms will not be discussed here.

As an efficient example of signal stripping approach, we can reconstruct each user's channel matrix individually and use it for sensing

$$
\hat{\mathbf{H}}_{n, k, t}=\mathbf{H}_{n, k, t}+\boldsymbol{\Delta}_{n, k, t},
$$

where $\boldsymbol{\Delta}_{n, k, t}$ is the reconstruction error.

To reduce clutter in the estimation, we can apply differential techniques by computing the difference between $\hat{\mathbf{H}}_{n, k, t}$ and $\hat{\mathbf{H}}_{n, k, t+T}$, and obtain

$$
\begin{aligned}
& \hat{\mathbf{H}}_{n, k, t+T}-\hat{\mathbf{H}}_{n, k, t} \\
= & \sum_{\ell=1}^{L_{k}} b_{k, \ell} e^{-j 2 \pi n \tau_{k, t, \ell} f_{0}} e^{j 2 \pi f_{D, k, t, \ell} T_{s}(t-T / 2)} \\
& \left(-2 \sin \left(\pi f_{D, k, t, \ell} T T_{s}\right)\right) \mathbf{a}\left(M, \phi_{k, t, \ell}\right) \mathbf{a}^{T}\left(M_{T}, \theta_{k, t, \ell}\right)+ \\
& \mathbf{H}_{n, k, t+T}(\text { new })+\boldsymbol{\Delta}_{n, k, t+T}+\boldsymbol{\Delta}_{n, k, t}
\end{aligned}
$$

where $\mathbf{H}_{n, k, t+T}$ (new) denotes terms associated with new multipath signals at time $t+T$.

When both transmitters and receivers are static, (5) indicates that only moving objects in the environment will be seen in the differential signal, and clutter from static objects will be removed. The expression is similar to that in (3) with potentially significantly reduced multipath numbers, while the term $\left(-2 \sin \left(\pi f_{D, k, t, \ell} T T_{s}\right)\right)$ can be absorbed into $b_{k, t, \ell}$. This will hence be particularly useful in downlink sensing where all BSs are static. In uplink sensing, when a transmitting user is moving, sensing based on differential signals can still be useful through applying multiple different values for $T$. In this way, signals with different Doppler shifts can be filtered out by using different $T_{\mathrm{s}}$ in differential signals, and parameter estimation can be combined with recursive multipath cancellation. Detailed development is beyond the scope of this paper.

\subsection{Delay-Quantized On-grid Formulation}

In this paper, we propose 1-D compressive sensing based algorithms for sensing parameter estimation using the reconstructed channel model in (4). Since the differential signal model in $(5)$ is similar to that in (3), we use (3) as a generalized channel matrix 
model, and drop the subscripts $t$ and $k$ in the parameter variables. Assume $N_{u} \gg L$. We consider an on-grid delay model where the delays $\tau_{\ell} f_{0}$ are quantized as $q_{\ell} / N^{\prime}$ with $q_{\ell}$ being an integer. Therefore $e^{-j 2 \pi n \tau_{\ell} f_{0}} \approx e^{-j 2 \pi n q_{\ell} / N^{\prime}}$. Here, $N^{\prime}$ can equal to or be multiple times of $N$. The minimal resolvable delay is then $N /\left(N^{\prime} B\right)$.

We can now convert (3) to a delay-quantized model. For simplicity, we assume that there is only one multipath signal within each quantized delay bin. Referring to (3), the delay-on-grid model can be represented as

$$
\begin{gathered}
\mathbf{H}_{n}=\sum_{\ell=1}^{L} b_{\ell} e^{-j 2 \pi n q_{\ell} / N^{\prime}} e^{j 2 \pi t f_{D, \ell} T_{s}} . \\
\mathbf{a}\left(M, \phi_{\ell}\right) \mathbf{a}^{T}\left(M_{T}, \theta_{\ell}\right) \\
=\mathbf{A}_{R} \mathbf{D C}_{n} \mathbf{A}_{T}^{T},
\end{gathered}
$$

where the $\ell$-th column in $\mathbf{A}_{R}$ (or $\left.\mathbf{A}_{T}\right)$ is $\mathbf{a}\left(M, \phi_{\ell}\right)$ (or $\mathbf{a}\left(M_{T}, \theta_{\ell}\right)$ ), $\mathbf{D}$ and $\mathbf{C}_{n}$ are diagonal matrices with the $\ell$-th diagonal element being $b_{\ell} e^{j 2 \pi t f_{D, \ell} T_{s}}$ and $e^{-j 2 \pi n q_{\ell} / N^{\prime}}$, respectively.

When the number of antennas is small and $M<L$, it is impossible to directly get the estimate of sensing parameters using reconstructed per-subcarrier channel matrix. However, it is generally true that the number of used subcarriers is much larger than $L$. Hence we can formulate (6) as a 1-D CS problem and get the estimates for $q_{\ell} \mathrm{S}$ first. The $m$-th column of $\mathbf{H}_{n}$ can be represented as

$$
\mathbf{h}_{n, m}=\mathbf{A}_{R} \mathbf{D} \mathbf{P}_{m} \mathbf{c}_{n},
$$

where $\mathbf{P}_{m}$ is a diagonal matrix with diagonal elements being the $m$-th row of $\mathbf{A}_{T}$, and $\mathbf{c}_{n}$ is an $L \times 1$ vector with the $\ell$-th element $e^{-j 2 \pi n q_{\ell} / N^{\prime}}$.

We may have a few options to process different columns of $\mathbf{H}_{n}$. For the least, we need two columns so that $\mathrm{AoD}$ can be estimated. Use $M_{t}=2$ as a simple example. Transpose $\mathbf{h}_{n, m}, m=1,2$ and stack them into a row vector

$$
\left(\mathbf{h}_{n, 1}^{T}, \mathbf{h}_{n, 2}^{T}\right)=\mathbf{c}_{n}^{T} \mathbf{D}\left(\mathbf{P}_{1} \mathbf{A}_{R}^{T}, \mathbf{P}_{2} \mathbf{A}_{R}^{T}\right) .
$$

Now stacking similarly formulated row vectors for all usable subcarriers $n \in \mathcal{S}$ together, we can obtain

$$
\widetilde{\mathbf{H}}=\mathbf{W} \underbrace{\mathbf{D}\left(\mathbf{P}_{1} \mathbf{A}_{R}^{T}, \mathbf{P}_{2} \mathbf{A}_{R}^{T}\right)}_{\boldsymbol{G}},
$$

where the $\ell$-th column of the $N_{u} \times L$ matrix $\mathbf{W}$ is $\left\{e^{-j 2 \pi n q_{\ell} / N^{\prime}}\right\}, n \in \mathcal{S}$.

\subsection{Estimation of Sensing Parameters}

We now convert the multipath signal model in (9) to a generalized delay-on-grid sparse model, by representing it using $N_{p} \gg L, N_{p} \leq N^{\prime}$ multipath signals where only $L$ signals are non-zeros. Without any prior knowledge of the delay, we can use $N_{p}=N^{\prime}$; otherwise, the range of delays can be reduced. We can then treat it as a multiple measurement vector (MMV) CS problem and use algorithms such as OMP or Bayesian CS to get the estimate for $\mathbf{W}$ and $\boldsymbol{G}$. The dictionary is a partial $N_{u} \times N_{p}$ DFT matrix F. When $N_{p}=N^{\prime}$, its $q$-th column is $\left\{e^{j 2 \pi n q / N^{\prime}}\right\}, n \in \mathcal{S}$.

Let $g_{\ell, p}$ be the $(\ell, p)$-th element of $\boldsymbol{G}$. Once the delays and $\boldsymbol{G}$ are estimated, we can get the estimates for AoA and AoD through

$$
2 \pi d \sin \left(\phi_{\ell}\right) / \lambda=\angle(\underbrace{\frac{1}{2 M} \sum_{k=0}^{1} \sum_{p=1}^{M-1} g_{\ell, p+k M}^{H} g_{\ell, p+1+k M}}_{\varepsilon_{\ell}}),
$$$$
2 \pi d \sin \left(\theta_{\ell}\right) / \lambda=\angle(\underbrace{\frac{1}{M} \sum_{p=1}^{M} g_{\ell, p}^{H} g_{\ell, p+M}}_{\xi_{\ell}}),
$$

respectively. The value of $\left|b_{\ell}\right|^{2}$ can also be obtained easily during the process of computing AoA and AoD, being either $\left|\varepsilon_{\ell}\right|^{2}$ or $\left|\xi_{\ell}\right|^{2}$. The estimates of $\left|b_{\ell}\right|^{2}$ can be used to find the effective multipath delay bins in noisy channels, by using a threshold determined, e.g., as a fractional scalar of the maximum power of multipath signals.

The computation above can be readily extended to the case when $M_{T}>2$.

This process can be repeated over multiple OFDM symbols. The Doppler shift can then be estimated from the cross-correlation between two or more $\boldsymbol{G}$ s that are sufficiently spaced in time, while channel still remains stable except for the Doppler phase terms. Let $\boldsymbol{G}_{t}$ denote the estimate of $\boldsymbol{G}$ at the $t$-th OFDM symbol. The estimates of Doppler shift can be obtained from

$$
2 \pi f_{D, \ell} T_{s}=\angle\left(\left(\boldsymbol{G}_{t+T}\right)_{\ell, \cdot}\left(\left(\boldsymbol{G}_{t}\right)_{\ell, \cdot}\right)^{H}\right),
$$

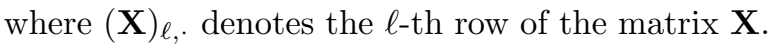

\section{SIMULATION RESULTS}

We present simulation results using the Bayesian Sparse Learning algorithm [4] to validate the effectiveness of our parameter estimation scheme. We consider a system with $4 \mathrm{BSs}$, each with a 8-element antenna array, providing connections to users each with 2 antennas. The interval between antennas is $d=\lambda / 2$. The number of subcarriers is $N=256$, and $8 \times 8$ multiuser MIMO over each subcarrier is applied. The carrier frequency is $2.35 \mathrm{GHz}$ and 

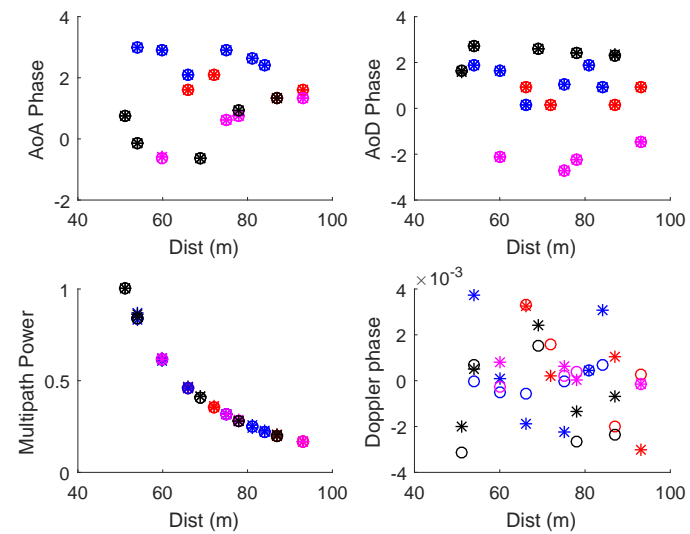

Figure 1: Parameter estimation for downlink sensing with $\eta=12 \mathrm{~dB}, T=4 T_{s}$ and $\gamma=0.1$. Different colours correspond to different BSs. Circles for actual values, and starts for estimated ones.

the signal bandwidth is $100 \mathrm{MHz}$. No radar crosssection information is considered. Multipath signals for each BS/user are generated randomly in cluster, mimicking reflected/scattered signals from objects. Each cluster is generated following uniform distributions of $[3,6]$ for the total multipath number, and AoAs, AoDs and Doppler frequencies are randomly generated within a given range. Delays are on grid with an interval of $10 \mathrm{~ns}$, corresponding to a distance resolution of $3 \mathrm{~m}$. Delays from the same BS/user are kept different. But they could be the same between BSs/users.

Channel reconstruction error as shown in (4) is introduced as AWGN. The ratio between the mean power of the channel coefficients and AWGN is denoted by $\eta$. A threshold of $\gamma \cdot \max \left(\operatorname{abs}\left(\xi_{\ell}\right)\right)$ is used to pick up effective multipath delay estimates from the MMV CS estimation output.

Fig. 1 shows the results for downlink sensing where all $N_{u}=N^{\prime}=256$ subcarriers are used. It can be seen that the estimates of delay, AoA, and AoD are accurate and is robust to the introduced channel reconstruction error. The estimate of Doppler phase has relatively large error because the actual Doppler phase values are very small and hence sensitive to noise. Wherever possible, larger $T$ shall be used for estimating Doppler shifts.

Fig. 2 demonstrates the results for uplink sensing where the indexes of the used $N_{u}=N^{\prime}=64$ subcarriers are randomly generated. Estimates in this case is more noisy and hence larger $\gamma$ is used, which also removes a few effective estimates (two circles in the figure without matching estimates). The estimation accuracy for Doppler phase is improved through the use of larger $T$ values.
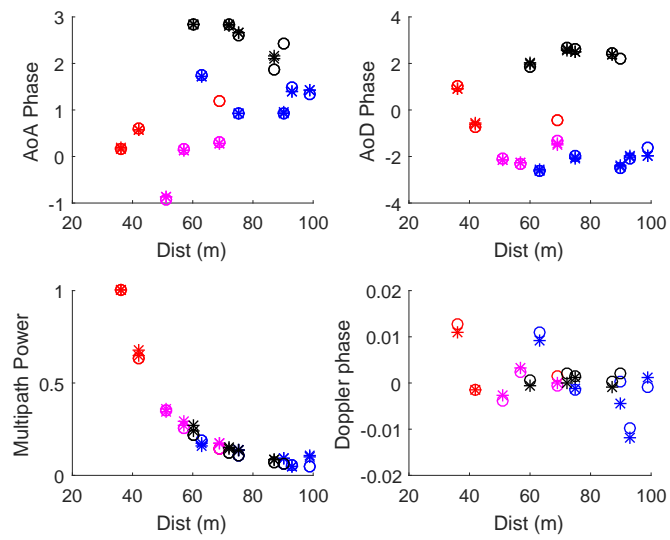

Figure 2: Parameter estimation for uplink sensing with $\eta=12 \mathrm{~dB}, T=10 T_{s}$ and $\gamma=0.25$. Different colours correspond to different users.

\section{CONCLUSIONS}

We have demonstrated that by using signal stripping approaches and reconstructing the channel matrix we can simplify the signal model used for sensing parameter estimation for both uplink and downlink sensing in the recently proposed perceptive mobile networks. Conventional 1-D compressive sensing algorithms can then be used to estimate propagation delays. We also developed methods for estimating all of the other sensing parameters. Simulation results demonstrate that all methods work effectively and are robust to the channel reconstruction error.

\section{References}

[1] J. A. Zhang, A. Cantoni, X. Huang, Y. J. Guo, and R. W. H. Jr., "Development of perceptive mobile network using joint communications and sensing," in VTC Spring 2017, June 2017.

[2] C. Sturm and W. Wiesbeck, "Waveform design and signal processing aspects for fusion of wireless communications and radar sensing," Proceedings of the IEEE, vol. 99, no. 7, pp. 12361259, July 2011.

[3] O. O. Oyerinde and S. H. Mneney, "Subspace tracking-based decision directed cir estimator and adaptive cir prediction," IEEE Transactions on Vehicular Technology, vol. 61, no. 5, pp. 2097-2107, Jun 2012.

[4] S. Ji, Y. Xue, and L. Carin, "Bayesian compressive sensing," Signal Processing, IEEE Transactions on, vol. 56, no. 6, pp. 2346-2356, June 2008. 